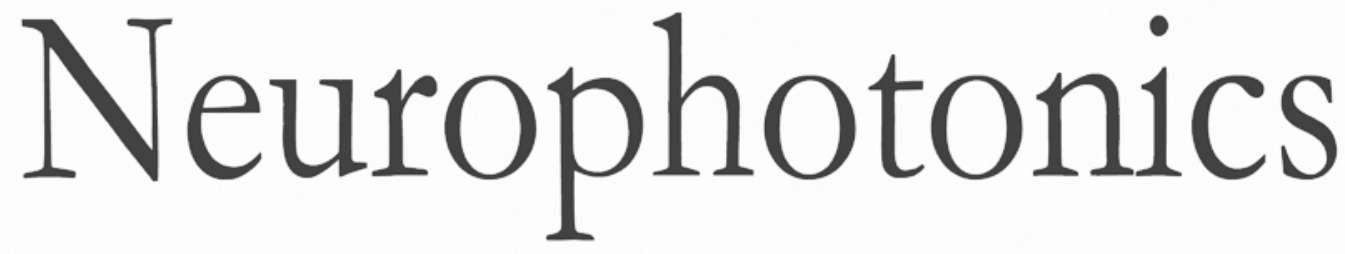

\title{
Errata: Quantum mechanism of light transmission by the intermediate filaments in some specialized optically transparent cells
}

\author{
Vladimir Makarov \\ Lidia Zueva \\ Tatiana Golubeva \\ Elena Korneeva \\ Igor Khmelinskii \\ Mikhail Inyushin
}




\title{
Errata: Quantum mechanism of light transmission by the intermediate filaments in some specialized optically transparent cells
}

\author{
Vladimir Makarov, ${ }^{a}$ Lidia Zueva, ${ }^{b}$ Tatiana Golubeva, ${ }^{c}$ Elena Korneeva, ${ }^{d}$ Igor Khmelinskii, ${ }^{e}$ and Mikhail Inyushin \\ aUniversity of Puerto Rico, Department of Physics, Rio Piedras Campus, P.O. Box 23343, San Juan 00931-3343, Puerto Rico \\ ${ }^{\mathrm{b}}$ Russian Academy of Sciences, Sechenov Institute of Evolutionary Physiology and Biochemistry, St. Petersburg, Russia \\ 'Lomonosov State University, Department of Vertebrate Zoology, Moscow 119992, Russia \\ ${ }^{\mathrm{d} R u s s i a n}$ Academy of Sciences, Institute of Higher Nervous Activity and Neurophysiology, Butlerova Street 5a, Moscow 117485, Russia \\ eUniversidade do Algarve, Centro de Investigação em Química do Algarve (CIQA), Faro 8005-139, Portugal \\ fUniversidad Central del Caribe, School of Medicine, Department of Physiology, Bayamón 00960-6032, Puerto Rico
}

[DOI: 10.1117/1.NPh.4.1.019801]

This article [Neurophoton. 4(1), 011005 (2016).] was originally published online on 16 Aug 2016 with an error in the Introduction on p. 1. The original text read:

"However, the guinea pig retina contains a regular pattern of MCs arranged mostly in parallel to each other, spanning the entire thickness of the retina ( $\approx 500$ to $800 \mu \mathrm{m}$ )."
The text has been changed to read:

"However, the guinea pig retina contains a regular pattern of MCs arranged mostly in parallel to each other, spanning the entire thickness of the retina ( $\approx 120$ to $150 \mu \mathrm{m})$."

The article was corrected online on 30 August 2016. It appears correctly in print. 